\title{
Cardiovascular outcomes following percutaneous coronary intervention with drug-eluting balloons in chronic kidney disease: a retrospective analysis
}

\author{
Michael Jonas ${ }^{1 \dagger}$, Maayan Kagan ${ }^{2 \dagger}$, Gal Sella ${ }^{1}$, Dan Haberman ${ }^{1}$ and Gil Chernin ${ }^{2 *}$ (D)
}

\begin{abstract}
Background: Chronic kidney disease (CKD) is associated with poorer outcomes following percutaneous coronary intervention ( $\mathrm{PCl}$ ) with drug-eluting stents. Drug-eluting balloons are used for in-stent restenosis and selected cases of de-novo coronary lesions. Little is known regarding the outcomes of individuals with CKD who undergo PCI with drug-eluting balloons. The goal of this study was to assess outcomes of $\mathrm{PCl}$ with drug-eluting balloons in individuals with CKD.

Methods: In a retrospective analysis, outcomes of $\mathrm{PCl}$ with drug-eluting balloons were compared between 101 patients with CKD and 261 without CKD. CKD was defined as estimated glomerular filtration rate $<60 \mathrm{ml} / \mathrm{min} /$ $1.73 \mathrm{~m}^{2}$. We compared demographics, procedure data and clinical outcomes in the first and second years following the procedure.

Results: Rates of major adverse cardiac events (MACE) and myocardial infarction were higher in patients with than without CKD: $23.8 \%$ vs. $13.8 \%, P<0.005$ and $15.9 \%$ vs. 3.8\%, $P<0.001$, respectively. Rates of target lesion revascularization were similar, 14.9 and $11.5 \%$, respectively, $P=0.4$. Shorter duration of dual anti-platelet therapy was observed among patients with than without CKD $(10.0+3.4$ vs. $10.9+3.7$ months, $P<0.05)$. First-year hemorrhage episodes were similar in the two groups ( $0.08 \pm 0.4$ and $0.03 \pm 0.2$, respectively, $P=0.2$ ). In a multivariate regression analysis, $C K D$ was associated with increased risks of first year MACE (OR 2.1; 95\% confidence interval 1.0-4.3, $P<0.001$ ).

Conclusions: $\mathrm{PCl}$ with drug-eluting balloons was associated with increased cardiovascular morbidity and mortality in patients with than without CKD. However, rates of target lesion revascularization were similar in the two groups. Shorter duration of dual anti-platelet therapy was observed in the CKD group.
\end{abstract}

Keywords: Drug-eluting stents, Chronic kidney disease, Drug-eluting balloons, Percutaneous coronary intervention, Dual anti-platelet therapy, Cardiovascular mortality, In-stent restenosis, Ischemic heart disease, Target lesion revascularization

\footnotetext{
* Correspondence: Chernin4@gmail.com

${ }^{\dagger}$ Michael Jonas and Maayan Kagan contributed equally to this work. ${ }^{2}$ Department of Nephrology and Hypertension, Kaplan Medical Center, Hebrew University School of Medicine, Pasternak St. POB1, 76100 Rehovot, Israel

Full list of author information is available at the end of the article
}

(c) The Author(s). 2020 Open Access This article is licensed under a Creative Commons Attribution 4.0 International License, which permits use, sharing, adaptation, distribution and reproduction in any medium or format, as long as you give appropriate credit to the original author(s) and the source, provide a link to the Creative Commons licence, and indicate if changes were made. The images or other third party material in this article are included in the article's Creative Commons licence, unless indicated otherwise in a credit line to the material. If material is not included in the article's Creative Commons licence and your intended use is not permitted by statutory regulation or exceeds the permitted use, you will need to obtain permission directly from the copyright holder. To view a copy of this licence, visit http://creativecommons.org/licenses/by/4.0/ The Creative Commons Public Domain Dedication waiver (http://creativecommons.org/publicdomain/zero/1.0/) applies to the data made available in this article, unless otherwise stated in a credit line to the data. 


\section{Background}

Percutaneous coronary intervention (PCI) with drugeluting balloons (DEB) was established as an effective treatment for patients with ischemic heart disease (IHD) and in-stent restenosis (ISR) [1, 2]. In selected patients with IHD, DEBs are also used for de-novo lesions, though small randomized control trials reported conflicting results in this regard [1, 3, 4]. DEBs mechanically open obstructed vessels by inflating; this releases a homogeneous high dose of anti-proliferative drug (such as paclitaxel). The resultant reduction in neointimal proliferation prevents restenosis of the obstructed vessel [2, 5].

Unlike the commonly used drug-eluting stents (DES), DEB angioplasty does not entail implantation of a foreign body in the blood vessel. Thus, a shorter period of dual anti-platelet therapy (DAPT) is required, since it is conceivable that DEB does not confer a risk for stent thrombosis. A shorter period of DAPT could be beneficial for patients with bleeding diathesis, such as patients with chronic kidney disease (CKD) $[2,6]$.

Renal dysfunction is a known risk factor for IHD, and is associated with accelerated atherosclerosis, endothelial dysfunction, oxidative stress and inflammation. These contribute to the formation of coronary artery atherosclerosis and increased cardiovascular mortality [7-9]. In addition, individuals with CKD who underwent PCI with DES were shown to have poorer post-procedural cardiovascular outcomes than patients without CKD, including higher mortality, hemorrhagic complications and prolonged hospitalizations $[9,10]$.

Little is known about DEB angioplasty outcomes among patients with CKD. Data from the Korean Multicenter In-Stent Restenosis Registry suggest that while rates of target lesion failure were lower in contemporary DES compared with DEB, this difference was attenuated in patients with CKD (defined as an estimated glomerular filtration rate $(\mathrm{eGFR})<60 / \mathrm{ml} / \mathrm{min} / 1.73 \mathrm{~m} 2$ ) [11]. The aim of the current study was to compare outcomes of patients who underwent PCI with DEB, between those with and without CKD.

\section{Methods}

This study was approved by the Institutional Review Board of the Kaplan Medical Center. We performed a retrospective, all comers, single center registry of patients who underwent DEB angioplasty between August 2011 and October 2017. Electronic medical records of enrolled patients were obtained from the electronic system of the Kaplan Medical Center and from the 'Ofek' system, the medical record system of the Clalit Health Services, the largest health provider in Israel.

Patients meeting the inclusion criteria were males and non-pregnant females, older than 18 years, who attended regular follow-up with cardiology consultants at least every 6 months after the procedure, and who had at least two creatinine serum level tests. The first of these tests was three to 6 months prior to the procedure, and the second test in the 3 months before the PCI with DEB angioplasty. Clinical indications for angiography included acute coronary syndrome (ACS), angina pectoris, congestive heart failure (CHF) or arrythmia evaluation, or a positive stress test. Patients with acute kidney injury, as defined by the KDIGO criteria [12], or a recent myocardial infarction (MI) defined as $\leq 7$ days of PCI were excluded from the analysis. The decision to use DEB was made by individual operators under considerations of lesion complexity, the inability to deliver a stent or the requirement for a shorter DAPT regimen (either due to bleeding tendencies or the need to temporarily stop DAPT for planned non-vascular invasive procedures).

For each patient who met the eligibility criteria we reviewed baseline characteristics including age, gender, body mass index and ejection fraction measured in the year before the PCI. Additionally, we reviewed background conditions such as CHF, diabetes mellitus, chronic obstructive pulmonary disease, hypertension and hyperlipidemia.

\section{Definition of variables, measurements and procedure characteristics}

Creatinine baseline was defined as the closest measurement prior to the procedure, within 3 months. For each patient, we used the baseline measurement to calculate eGFR with the CKD-EPI formula [13]. Creatinine assay in this report used methods that are traceable to isotope dilution mass spectrometry (IDMS) reference methodology. The cohort was stratified according to the absence or presence of CKD, defined as eGFR $<60 / \mathrm{ml} / \mathrm{min} /$ $1.73 \mathrm{~m}^{2}$ for more than 3 months. The eGFR was calculated according to the last creatinine before the PCI ".

We assessed the characteristics of the angioplasty including the indication of the catheterization (ISR or denovo), the target vessel (right coronary artery, left main coronary artery, left anterior descending artery, left circumflex or graft vessel), the type of DEB used in the procedure, the number of balloons used during the procedure, the length and size of the lesion, the type of DAPT that was recommended following the procedure (Clopidogrel, Ticagrelor or Prasugrel) and the duration of DAPT following the procedure. The duration of DAPT was analyzed both continuously and categorically (categories were defined as below or above 6 months).

The DEBs that were used in the procedures, at a dose of $3.0 \mu \mathrm{g} / \mathrm{mm}^{2}$ of paclitaxel, were 'Pantera Lux' (Biotronik, Bülach, Switzerland) or 'SeQuent Please' (B. Braun, Melsungen AG, Berlin, Germany) or both. Additionally, some of the procedures used 'AngioSculpt' by Biotronik. 
Seventeen patients underwent two procedures during the registry years. For each of these patients, we analyzed data from the first procedure and noted the additional procedure as an outcome.

For twelve patients, an intervention was performed in more than one vessel during the PCI. We excluded these patients from the analysis of vessel type, lesion length and lesion size. These parameters were included in the regression analysis.

\section{Outcomes}

The main outcome was major adverse cardiac event (MACE) during the first year, defined as the composite of three endpoints: cardiac hospitalizations, cardiac death and target lesion revascularization (TLR). Cardiac hospitalizations were defined as hospitalizations due to one of the following: MI, ACS, chest pain (without an alternative non-cardiac diagnosis), arrhythmia or heart failure exacerbation. Cardiac death was defined as death due to one of the above cardiac hospitalization etiologies. Additional analyses of first and second-year cardiac death and cardiac hospitalizations were obtained. We also analyzed first year hemorrhages, defined as type 2 or higher bleeding events according to the BARC criteria [14].

\section{Statistical analysis}

Continuous variables were described using means \pm standard deviations; categorical variables were described by frequencies and percentages. The Chi square test, $\mathrm{T}$ test and Fisher's exact test were used to calculate differences between the groups, as appropriate. A multivariant regression analysis was used to determine the independent effect of CKD on the outcomes examined. The Kaplan-Meier plot was used to estimate survival in the first year of follow up using the log-rank test. All statistical analyses were performed with SAS version 9.4 (SAS Institute, Cary, North Carolina).

\section{Results}

Of 362 patients who met the inclusion criteria, 101 had CKD, defined as baseline eGFR $<60 \mathrm{ml} / \mathrm{min} / 1.73 \mathrm{~m}^{2}$. Baseline characteristics are summarized in Table 1. Two patients were excluded from the analysis because of lack of first creatinine levels three to 6 months prior to the procedure. The mean eGFR for the CKD and non-CKD groups were $40.2 \pm 17.9$ and $86.1 \pm 17.4 \mathrm{ml} / \mathrm{min} / 1.73 \mathrm{~m}^{2}$, respectively. Of the 101 patients with CKD, 52 had CKD stage 3A (eGFR $45-59 \mathrm{ml} / \mathrm{min} / 1.73 \mathrm{~m} 2), 25$ had CKD stage 3B (eGFR $30-44 \mathrm{ml} / \mathrm{min} / 1.73 \mathrm{~m} 2$ ), and 24 had CKD stage 4 (eGFR $15-29 \mathrm{ml} / \mathrm{min} / 1.73 \mathrm{~m} 2)$.

Compared to patients without CKD, among those with CKD, the mean age was older $(72.5+10.8$ versus $64.0+$ 10.8 years, $P<0.001)$ and the proportion of males was smaller $(71.3 \%$ vs. $83.9 \%, P<0.01)$. Additionally, the
CKD group had substantially higher rates of comorbidities such as diabetes mellitus $(60.4 \%$ vs. $40.2 \%, P<$ $0.001)$, hypertension $(89.1 \%$ vs. $66.5 \%, P<0.001)$ and CHF (13.9\% vs. $1.5 \%, P<0.001)$; and a lower rate of smoking $(27.7 \%$ vs. $42.9 \%, P<0.01)$ (Table 1$)$.

Of 261 patients with PCI with DEB due to de-novo lesions, 65 (25\%) had CKD (Table 1). Compared to patients without CKD, those with CKD were older and more likely with diabetes, $\mathrm{CHF}$ and hypertension; and less likely smokers.

Of the 101 patients who underwent PCI with DEB due to ISR, 97 had restenosis in DES, 3 patients in bare metal stents, and the type of stent was unknown in one patient. Thirty-six (36\%) of these patients had CKD. Compared to those without CKD, the mean age of those with CKD was older (Table 1), and the proportion with CHF was higher.

\section{Procedure characteristics, lesion morphology and antiplatelet therapy}

Angiographic data and procedure characteristics are presented in Table 2. De-novo catheterization, compared to ISR, was the indication for PCI in a lower proportion in the CKD than the non-CKD group $(64.4 \%$ vs. $75.1 \%$, $P<0.05)$. The proportions of procedures that were ambulatory rather than urgent were similar between the groups. Lesion size and length were similar for the CKD group (mean $2.7 \pm 0.6 \mathrm{~mm}$ and $22.5 \pm 6.4 \mathrm{~mm}$, respectively) and the non-CKD group (mean $2.6 \pm 0.6 \mathrm{~mm}$ and $22.7 \pm 6.5 \mathrm{~mm}$, respectively). The number of balloons used was similar in the two groups (mean $1.1 \pm 0.4$ and $1.1 \pm 0.2$, respectively, $P=0.1$ ) (Table 2). The types of DEB ('Pantera Lux' vs. 'SeQuent Please' vs. both) did not differ significantly, according to the presence of CKD. The usage of both DEBs vs. Pantera Lux alone was higher for the CKD than the non-CKD group (15.5\% vs. $7.5 \%, P<0.05$ ).

For the patients with CKD, DAPT therapy with Prasugrel or Ticagrelor was less often implemented than was DAPT with Clopidogrel $(76.0 \%$ vs. $24.0 \%, P<0.01)$. Shorter duration of DAPT was observed among patients with than without CKD $(10.0 \pm 3.4$ vs. $10.9 \pm 3.7$ months, $P<0.05)$. Among those who underwent an ambulatory procedure, the duration of DAPT was shorter for those with than without CKD $(9.6 \pm 3.3$ vs. $11.3 \pm 4.6$ months, $P<0.05$ ).

In the sub-analysis of the de-novo cohort, lesion size and length were similar between those with and without CKD. The recommendation of DAPT with Prasugrel or Ticagrelor was substantially lower for patients with than without CKD (13.9\% vs. $32.7 \%, P<0.005)$, and the usage of both DEBs, vs. Pantera lux alone or vs. SeQuent Please alone, was greater $(20.6 \%$ vs. $7.3 \%, P=0.01)$. The duration of DAPT was shorter for the CKD group 


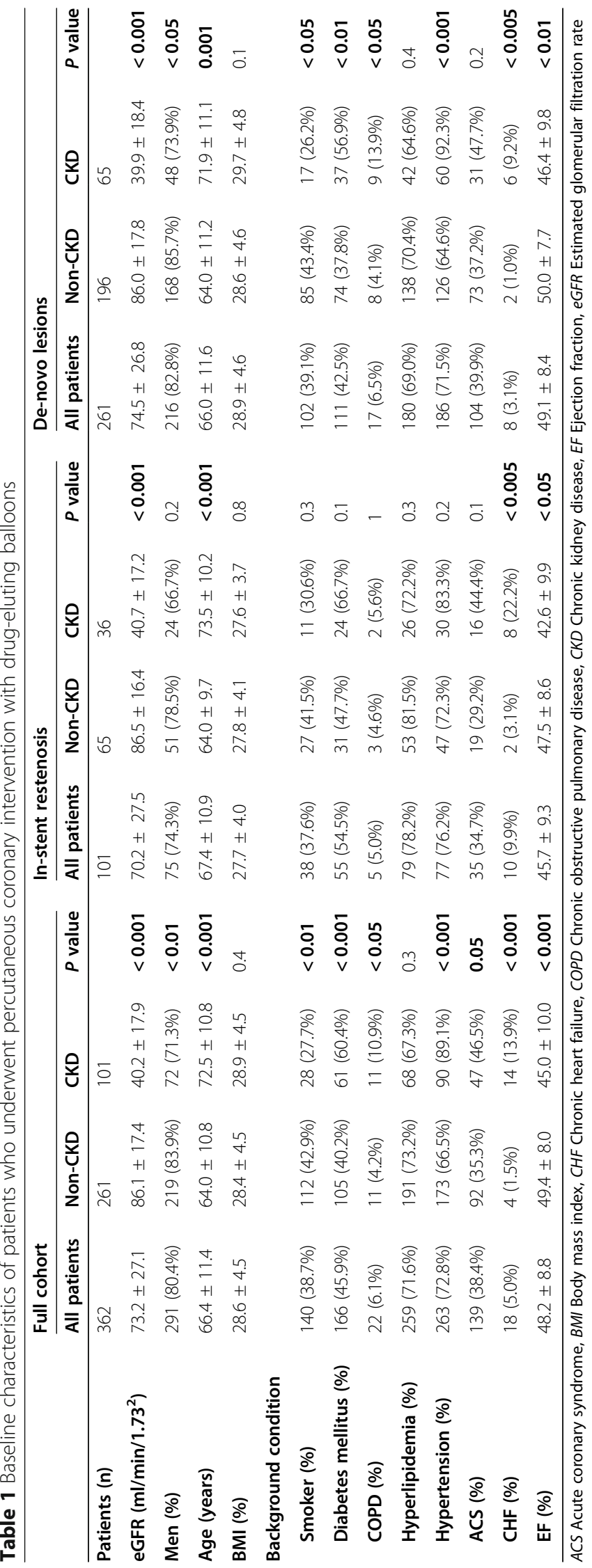




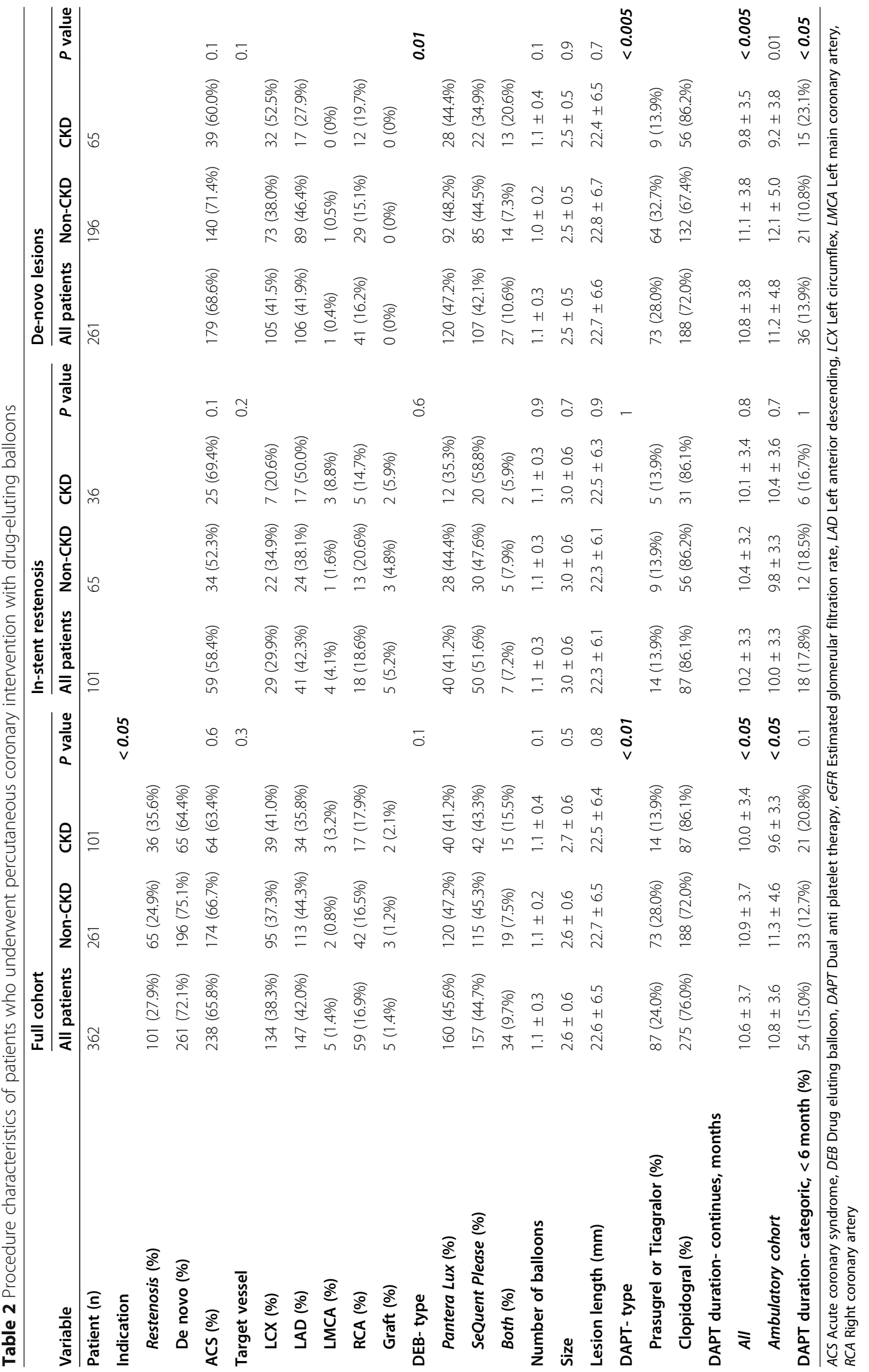


(9.8 \pm 3.5 vs. $11.1 \pm 3.8, P<0.005)$ (Table 2$)$. In the ISR cohort, lesion size and length, DAPT type, DAPT duration and DEB type were similar between the patients with and without CKD (Table 2).

\section{Outcomes}

Outcomes for the first and second years of follow up are summarized in Tables 3 and 4, and Fig. 1. MACE rates after 1 year were significantly higher for the CKD group (23.8\% vs. $13.8 \%, P<0.005)$. Compared to patients without CKD, for those with CKD, rates were higher of first year MI $(15.9 \%$ vs. $3.8 \%, P<0.001)$ and all-cause death (12.9\% vs. $2.3 \%, P<0.001)$ (Fig. 1$)$. Moreover, the CKD group had significantly higher rates of all-cause hospitalizations during the first month $(0.3 \pm 1.0$ vs. $0.1 \pm 0.4$, $P<0.05)$ and first year $(1.9 \pm 3.0$ vs. $0.8 \pm 1.4, P=0.001)$. Cardiac hospitalizations were also more common in the CKD group during the first year $(1.1 \pm 1.7$ vs. $0.5 \pm 1.0$, $P<0.005$ ) (Table 3). The groups were comparable for first year TLR, cerebrovascular accidents and major hemorrhagic events (Table 3).

Among patients who underwent PCI with DEB for ISR, higher rates of MACE $(38.9 \%$ vs. $16.9 \%, P<0.05)$ and MI $(30.6 \%$ vs. $7.7 \%, P<0.01)$ were noted in those with than without non-CKD (Table 3). Among patients who underwent PCI with DEB for de-novo lesions, rates of MACE and MI were not statistically different between CKD and non-CKD patients (Table 3).

In a multivariate regression model (linear and logistic, as appropriate), CKD was associated with increased risks of first year MACE (OR 2.1; 95\% confidence interval 1.04.3, $P<0.001)$ and MI (OR 4.8; 95\% confidence interval 1.7- 14.2, $P<0.005$ ) (Table 4).

\section{Discussion}

In this study of patients who underwent PCI with DEB, rates of MACE, MI, cardiac and all-cause hospitalizations, and cardiac and all-cause death were higher among those with CKD, defined as eGFR $<60 \mathrm{ml} / \mathrm{min} /$ $1.73 \mathrm{~m}^{2}$. To the best of our knowledge, this observational study is the first to thoroughly detail the outcomes of patients with CKD who underwent PCI with DEB. Nguyen et al found an association of CKD with increased MACE in patients treated with DEB for ISR. Yet, that study comprised a small number of patients with CKD, and CKD was not clearly defined [15].

Patients with CKD in the current study were older and had more risk factors associated with cardiac events and mortality, such as hypertension, heart failure and diabetes mellitus. Indeed, in this report, the increased age and the traditional risk factors contributed significantly to the adverse cardiovascular outcomes observed in the patients with CKD. In the multivariate regression analysis, CKD was significantly associated with cardiac hospitalizations, all-cause hospitalizations, and first and second-year all-cause death. Surprisingly, despite the poorer cardiovascular outcomes in the CKD group, the rates of TLR were similar for those with and without CKD. Due to the retrospective study design, we can only speculate regarding the underlying mechanisms leading to these rates. One possible explanation is that patients with CKD do not have higher rates of restenosis; this would imply that higher rates of cardiac events occur due to other mechanisms associated with CKD, such as arrhythmias, cardiac remodeling and heart failure [16]. These mechanisms are consequent to such changes as chronic volume overload, hyperactivation of the reninangiotensin system and erythropoietin deficiency anemia $[16,17]$. Another explanation for the similarity in TLR rates is physician's reluctance to catheterize patients with CKD due to the increased risk for complications following PCI $[18,19]$. Lower rates of catheterization could lead to both underestimation of lesion restenosis and poorer cardiovascular outcomes.

The association between CKD and cardiovascular disease is well established [16]. Indeed, cardiovascular disease is the most common cause of morbidity and mortality among CKD patients [16, 17, 20, 21]. CKD patients are more prone to IHD and tend to have poorer outcomes following revascularization with PCI or coronary artery bypass surgery $[8,22]$. Even in the era of PCI with the newer generation of DES, and with wellbalanced guideline-based medical therapy, adverse cardiovascular outcomes are more frequent in those with than without CKD $[10,19,23]$. Additionally, higher rates of MACE were recently reported in patients on hemodialysis who underwent PCI with DEB compared to patients not on hemodialysis [24]. Similarly, that study suggests an association of CKD with poorer outcomes following DEB angioplasty. However, in light of the potential benefits of DEB angioplasty for patients with CKD, outcomes of PCI with DEB versus DES should be investigated further, in selected coronary lesions.

Hypothetically, DEB angioplasty could be beneficial compared with the commonly used DES, for patients with a bleeding diathesis, such as those with CKD. This is because DEB enables a shorter period of DAPT, as short as 1-3 months (compared with the 12 month recommendation following DES implantation) [2, 6]. The current study showed a significantly shorter period of DAPT for the CKD group. However, while the minimal recommended time following DEB angioplasty is 1-3 months, the mean DAPT duration for the CKD group was $10.0 \pm 3.4$ months, suggesting that the full potential benefits of shorter DAPT was not realized. The DAPT recommendation following ACS is 12 months $[5,6]$. Thus, we performed an additional analysis of the patients who underwent an 


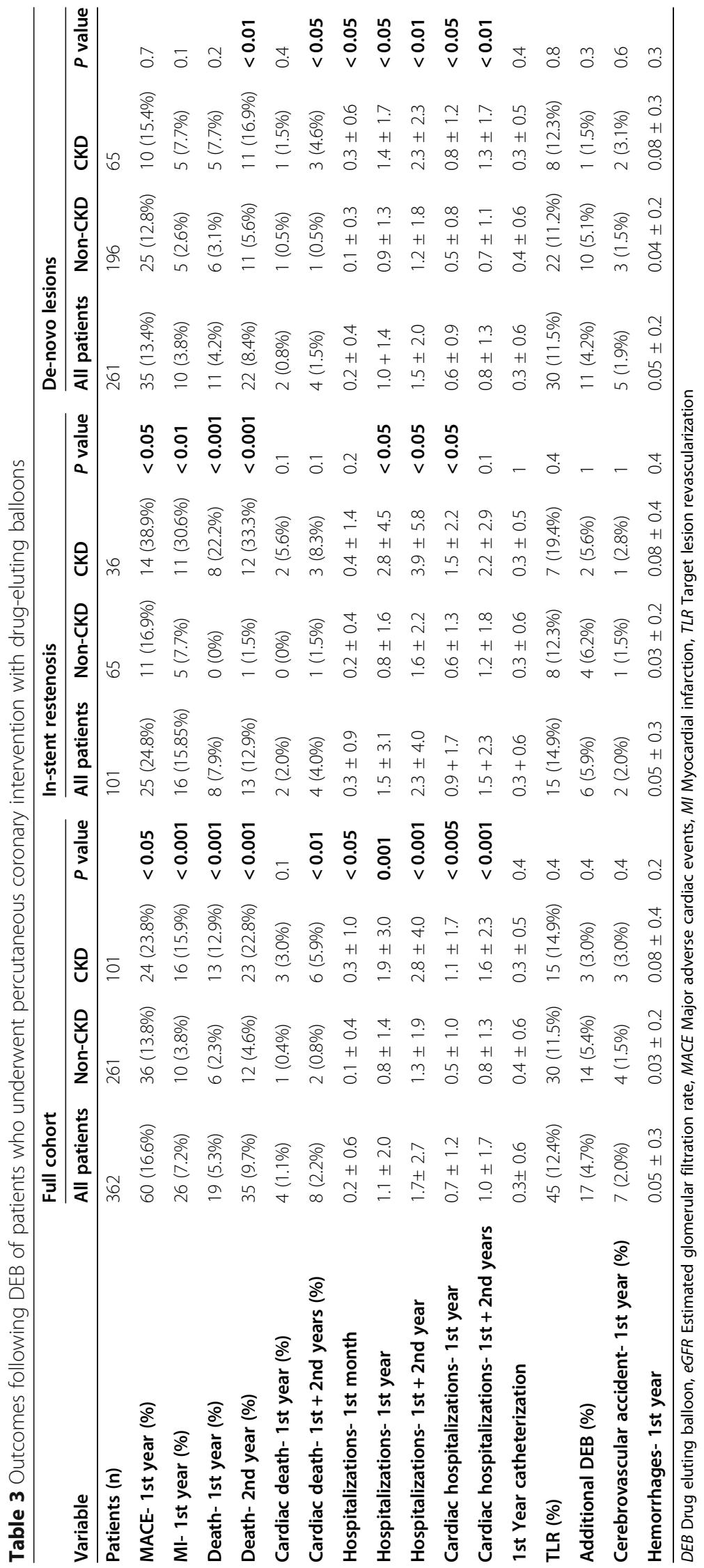


Table 4 Regression analysis of the independent effect of CKD on outcomes

\begin{tabular}{|c|c|c|c|c|c|c|c|c|}
\hline Variable & Coefficient & Cl 95\% & SD & OR & OR Cl 95\% & RR & RR Cl 95\% & $P$ value \\
\hline MACE-- 1st year & 0.7 & $0.02-1.5$ & 0.4 & 2.1 & $1.0-4.3$ & 1.8 & $0.9-3.3$ & $<0.05$ \\
\hline MI- 1st year & 1.6 & $0.5-2.6$ & 0.5 & 4.8 & $1.7-14.2$ & 3.6 & $1.4-9.1$ & $<0.005$ \\
\hline Death- 1st year & 1.1 & $(-0.2)-2.4$ & 0.7 & 3 & $0.9-11.1$ & 2.6 & $0.8-8.3$ & 0.1 \\
\hline Death- 2nd year & 0.9 & $0.03-1.9$ & 0.5 & 2.6 & $3.6-6.7$ & 2.2 & $0.9-5$ & $<0.05$ \\
\hline Cardiac death- 1st year & 41.3 & $(-33,096)-33,178.9$ & $16,845.8$ & 0 & 0-inf & 0 & 0-inf & 1 \\
\hline Cardiac death- $1 \mathrm{st}+2 \mathrm{nd}$ years & 0.8 & $(-1.3)-3.0$ & 1.1 & 2.3 & $0.3-20.0$ & 1.9 & $0.3-12.5$ & 0.5 \\
\hline Hospitalizations- 1st year & 0.7 & $0.2-1.2$ & 0.3 & & & & & 0.01 \\
\hline Hospitalizations- 1st + 2nd year & 1.1 & $0.4-1.8$ & 0.4 & & & & & $<0.05$ \\
\hline Cardiac hospitalizations- 1 st year & 0.5 & $0.1-0.8$ & 0.2 & & & & & $<0.01$ \\
\hline Cardiac hospitalizations- $1 \mathrm{st}+2 \mathrm{nd}$ years & 0.7 & $0.3-1.2$ & 0.2 & & & & & 0.001 \\
\hline 1st year catheterization & 0.01 & $(-0.2)-0.2$ & 0.08 & & & & & 0.9 \\
\hline TLR & 0.6 & $(-0.2)-1.4$ & 0.4 & 1.8 & $0.8-4$ & 1.6 & $0.8-3.4$ & 0.2 \\
\hline Additional DEB & $(-1.2)$ & $(-2.9)-0.5$ & 0.9 & 0.3 & $0.1-1.6$ & 0.3 & $0.1-1.6$ & 0.2 \\
\hline Stroke- 1st year & 0.3 & $(-2.0)-2.7$ & 1.2 & 1.4 & $0.1-14.3$ & 1.4 & $0.1-14.2$ & 0.8 \\
\hline Hemorrhages- 1st year & 0.02 & $(-0.05)-0.09$ & 0.04 & & & & & 0.6 \\
\hline
\end{tabular}

DEB Drug eluting balloon, MACE Major adverse cardiac events, MI Myocardial infraction, TLR Target lesion revascularization
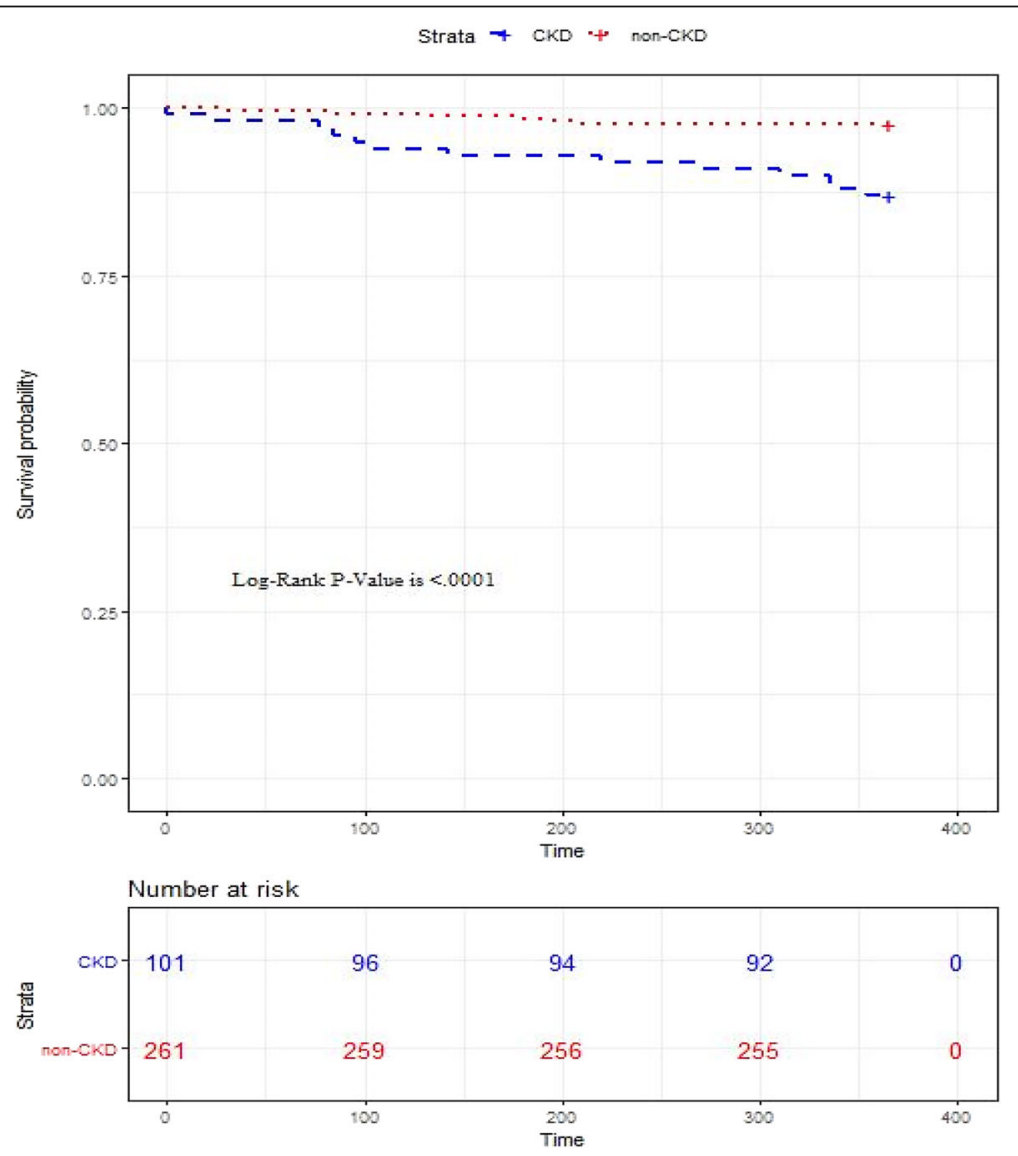

Fig. 1 Kaplan-Meier plot for one-year survival following percutaneous coronary interventions with drug-eluting balloons. CKD, Chronic Kidney Disease 
ambulatory procedure, to assess physicians' recommendation regarding DAPT, without the impact of ACS, which requires longer therapy. As expected, the DAPT duration was shorter for the CKD than the non-CKD group, yet still longer than the 1-3 months minimal required period. Furthermore, the difference between the CKD and the nonCKD groups in the ambulatory cohort was more substantial than in the full cohort. Interestingly, though the DAPT duration was longer than the minimally required duration, bleeding events were similar between the CKD and the non-CKD groups. Indeed, CKD patients may have a disrupted coagulation system and are prone to bleed following PCI with DAPT [22, 24]. These findings suggest that the shorter period of DAPT for the CKD group, though not fully exploited, may reduce bleeding events that could be life threatening [7]. Concurring with other studies, the rate of revascularization was lower among patients with than without CKD. One possible reason for such is the reluctance of physicians to catheterize patients with CKD due to complications such as bleeding [18, 22]. The option of shortening the DAPT duration should decrease concerns regarding long-term hemorrhagic events. Furthermore, recent studies showed that physician-guided cessation of DAPT after PCI with stenting is more frequent among patients with CKD, and this was shown to be associated with a higher rate of MACE [7]. Taken together, reducing the possible bleeding risk with shorter DAPT in patients with CKD may come at the expense of an increased risk for thrombotic events $[7,25]$. Therefore, randomized clinical trials are needed of patients with CKD, to address the minimal DAPT duration required following PCI.

This study has several limitations that should be acknowledged. First and foremost, as in any retrospective study, associations between CKD and poor outcomes following DEB angioplasty do not indicate causality. CKD status was defined by eGFR, based on creatinine measurements in the 3 months before PCI. It is therefore possible that some patients without CKD developed CKD in this interim and some patients with CKD may have experienced worsening of their disease. The decision to use DEB was made by the operator and as such, the probability of major selection bias exists. Intravascular ultrasound (IVUS) was not used routinely but rather in selected cases. Therefore, additional data of the detection by IVUS of coronary artery morphology is lacking. Additionally, while DAPT duration was shorter in the CKD patients, the observed duration was still longer than the minimal required time [2]. Indeed, not all patients with CKD had a shorter duration of DAPT and this may reflect other considerations for prolonged DAPT (e.g. concomitant peripheral vascular disease).

\section{Conclusions}

CKD was associated with adverse cardiovascular outcomes following PCI with DEB. Rates of TLR were similar for patients with and without CKD. Additional studies are needed to compare PCI with DEB versus DES, in the context of CKD, for the implementation of patient-tailored clinical recommendations.

\section{Abbreviations}

ACS: Acute coronary syndrome; CHF: Congestive heart failure; CKD: Chronic kidney disease; DAPT: Dual anti-platelet therapy; DES: Drug-eluting stents; eGFR: Estimated glomerular filtration rate; IHD: Ischemic heart disease; ISR: Instent restenosis; MACE: Major adverse cardiac event; PCl: Percutaneous coronary intervention; TLR: Target lesion revascularization

\section{Acknowledgments}

None.

\section{Authors' contributions}

MJ, MK and GC designed the study and drafted the manuscript. MK, GS and $\mathrm{DH}$ analyzed and interpreted the angiography data and substantively revised the work. All the authors read and approved the final manuscript.

\section{Funding}

This research did not receive any specific grant from funding agencies in the public, commercial, or not-for-profit sectors.

\section{Availability of data and materials}

The datasets generated and analyzed for the current study are not publicly available due to institute regulations, but they are available from the

corresponding author on reasonable request.

\section{Ethics approval and consent to participate}

The study was approved by the Ethics Committee of the Kaplan Medical Center. The Ethics Committee and the owner of the Kaplan Medical Center ('Clalit Health Services') granted administrative permissions to access the raw data. The need for consent for access to patient data was waived by the Ethics Committee.

Consent for publication

Not applicable.

\section{Competing interests}

The authors declare that they have no competing interests.

\section{Author details}

${ }^{1}$ Heart Institute, Kaplan Medical Center, Hebrew University School of Medicine, Rehovot, Israel. '2Department of Nephrology and Hypertension, Kaplan Medical Center, Hebrew University School of Medicine, Pasternak St. POB1, 76100 Rehovot, Israel.

Received: 22 May 2020 Accepted: 5 October 2020

Published online: 23 October 2020

\section{References}

1. Neumann FJ, Sousa-Uva M, Ahlsson A, et al. ESC/EACTS Guidelines on myocardial revascularization. Eur Heart J. 2018;40(2019):87-165.

2. Mohiaddin H, Wong TDFK, Burke-Gaffney A, Bogle RG. Drug-coated balloononly percutaneous coronary intervention for the treatment of De novo coronary artery disease: a systematic review. Cardiol Ther. 2018;7:127-49.

3. Rosenberg M, Waliszewski M, Chin K, et al. Prospective, large-scale multicenter trial for the use of drug-coated balloons in coronary lesions: the DCB-only all-comers registry. Catheter Cardiovasc Interv. 2019;93:181-8.

4. Fröhlich GM, Lansky AJ, Ko DT, et al. Drug eluting balloons for de novocoronary lesions - a systematic review and meta-analysis. BMC Med. 2013;11:123.

5. Her AY, Shin ES, Bang LH, et al. Drug-coated balloon treatment in coronary artery disease: recommendations from an Asia-Pacific consensus group. Cardiol J. 2019. https://doi.org/10.5603/CJ.a2019.0093.

6. Kleber FX, Rittger H, Bonaventura K, et al. Drug-coated balloons for treatment of coronary artery disease: updated recommendations from a consensus group. Clin Res Cardiol. 2013;102:785-97. 
7. Baber U, Li SX, Pinnelas R, et al. Incidence, patterns, and impact of dual antiplatelet therapy cessation among patients with and without chronic kidney disease undergoing percutaneous coronary intervention results from the Paris registry (patterns of non-adherence to anti-platelet regimens in stented patients). Circ Cardiovasc Interv. 2018;11:e006144.

8. Giustino G, Mehran R, Serruys PW, Sabik JF, Milojevic M, Simonton CA, et al. Left Main revascularization with $\mathrm{PCl}$ or CABG in patients with chronic kidney disease: EXCEL Trial. J Am Coll Cardiol. 2018;72:754-65.

9. Cai Q, Mukku V, Ahmad M. Coronary artery disease in patients with chronic kidney disease: a clinical update. Curr Cardiol Rev. 2013;9:331-9.

10. Gupta T, Paul N, Kolte D, et al. Association of chronic renal insufficiency with in-hospital outcomes after percutaneous coronary intervention. J Am Heart Assoc. 2015;4:e002069.

11. Lee JM, Rhee TM, Hahn JY, et al. Comparison of outcomes after treatment of in-stent restenosis using newer generation drug-eluting stents versus drug-eluting balloon: patient-level pooled analysis of Korean multicenter instent restenosis registry. Int J Cardiol. 2017;230:181-90.

12. KDIGO. Clinical Practice Guideline for Acute Kidney Injury. Kidney Int Suppl. 2012:2(Suppl 1):8.

13. Levey AS, Stevens LA, Schmid CH, et al. A new equation to estimate glomerular filtration rate. Ann Intern Med. 2009;150:604-12.

14. Mehran R, Rao SV, Bhatt DL, et al. Standardized bleeding definitions for cardiovascular clinical trials: a consensus report from the bleeding academic research consortium. Circulation. 2011;123:2736-47.

15. Nguyen VPT, Kim C, Hong SJ, et al. Comparison of clinical outcomes of two different types of paclitaxel-coated balloons for treatment of patients with coronary in-stent restenosis. Heart Vessel. 2019;34:1420-8.

16. McCullough PA. Why is chronic kidney disease the "spoiler" for cardiovascular outcomes? J Am Coll Cardiol. 2003;41:725-8.

17. Afsar B, Turkmen K, Covic A, Kanbay M. An update on coronary artery disease and chronic kidney disease. Int J Nephrol. 2014. https://doi.org/10. 1155/2014/767424.

18. Shavadia JS, Southern DA, James MT, Welsh RC, Bainey KR. Kidney function modifies the selection of treatment strategies and long-term survival in stable ischaemic heart disease: insights from the Alberta provincial project for outcomes assessment in coronary heart disease (APPROACH) registry. Eur Hear J Qual Care Clin Outcomes. 2018:4:274-82.

19. Chernin G, Chen S, Ozan O, et al. Percutaneous coronary intervention does not lower cardiovascular outcomes in patients with chronic kidney disease. Am J Nephrol. 2019;50:465-72.

20. Chronic Kidney Disease Prognosis Consortium, Matsushita K, van der Velde $M$, Astor B, et al. Association of estimated glomerular filtration rate and albuminuria with all-cause and cardiovascular mortality in general population cohorts: a collaborative meta-analysis. Lancet. 2010;375:2073-81.

21. Muntner P, He J, Astor BC, Folsom AR, Coresh J. Traditional and nontraditional risk factors predict coronary heart disease in chronic kidney disease: results from the atherosclerosis risk in communities study. J Am Soc Nephrol. 2005;16:529-38.

22. Rymer JA, Kaltenbach LA, Doll JA, Messenger JC, Peterson ED, Wang TY. Safety of dual-antiplatelet therapy after myocardial infarction among patients with chronic kidney disease. J Am Heart Assoc. 2019;8:e012236.

23. Scholz SS, Lauder L, Ewen S, Saarraaken K, Marx N, Sakhov O, et al. One-year clinical outcomes in patients with renal insufficiency after contemporary PCl: data from a multicenter registry. Clin Res Cardiol. 2019. https:/doi.org/ 10.1007/s00392-019-01575-y.

24. Kiriyama H, Kodera S, Minatsuki S, Kaneko H, Kikuchi H, Kiyosue A, et al. Short-term and long-term efficacy of drug-coated balloon for in-stent restenosis in hemodialysis patients with coronary artery disease. Int Heart J. 2019:60:1070-6.

25. Baber U, Mehran R, Giustino G, Cohen DJ, Henry TD, Sartori S, Ariti C, Litherland C, Dangas G, Gibson CM, Krucoff MW, Moliterno DJ, Kirtane AJ, Stone GW, Colombo A, Chieffo A, Kini AS, Witzenbichler B, Weisz G, Steg PG, Pocock S. Coronary thrombosis and major bleeding after $\mathrm{PCl}$ with drugeluting stents: Risk Scores From PARIS. J Am Coll Cardiol. 2016;67:2224-34. https://doi.org/10.1016/j.jacc.2016.02.064.

\section{Publisher's Note}

Springer Nature remains neutral with regard to jurisdictional claims in published maps and institutional affiliations. 